\title{
Negative Stain of Small Molecules and Protein Complexes
}

\section{Melissa Chambers}

\author{
Vanderbilt University, Nashville, Tennessee, United States
}

Negative stain of small molecules and protein complexes has useful applications to a range of biological studies. Negative stains are heavy metal solutions that significantly increase the contrast of the sample in the electron microscope and allow detail of the molecules to be visualized with a resolution limit of around $18 \AA$. Samples must be biochemically pure and conformationally homogeneous, preferably involving gel filtration as the final purification step. Each continuous carbon grid requires only $2.5 \mu 1$ of sample at a low concentration of $\sim 10 \mu \mathrm{g} / \mathrm{ml}$. A variety of stains can be used, with uranyl formate and uranyl acetate being best suited to small complexes. Most biological buffers are well tolerated with these stains, however it is preferable to avoid glycerol, phosphate (such as PBS) and high salt concentrations. There are also a variety of methods for applying stain and sample to the grids than can have an effect on the thickness and quality of the stain. Every sample is unique, and these methods are widely adaptable. Negative stain is a relatively quick way to assess the condition of the sample and ideal for troubleshooting purification and buffer conditions before continuing on with cryo-EM work. 2D class averages from negative stain images can reveal a lot about the homogeneity, composition, and flexibility of a complex, guiding the best conditions to be used when preparing vitrified grids for cryo-EM. In general, 2500-5000 individual particles are enough to yield informative results from negative stain 2D class averages, with less homogeneous samples requiring more particles to properly classify all conformations. In addition to being used for preliminary studies for cryo-EM, negative stain can be used to analyze homogeneity for crystallization trials and is well used in many non-structural applications. Class averages can be used to ensure full saturation of a complex with binding partners, paired with labeling techniques for domain identification, and as an extra step in verifying complex purification. Even single images can be informative for confirming proper reconstruction of virus-like particles and studying the effect of treatment on microtubule growth and catastrophe.

\section{References}

1. Negative Staining and Image Classification - Powerful Tools in Modern Electron Microscopy. Ohi M, Li Y, Cheng Y, Walz T. Biol Proced Online 2004; 6: 23-34.

2. Variations on Negative Stain Electron Microscopy Methods: Tools for Tackling Challenging Systems Scarff CA, Fuller, MJG, Thompson RF, Iadaza MG J Vis Exp 2018; (132): 57199. 